\title{
EDUCAÇÃO NUTRICIONAL COMO FORMA DE INTERVENÇÃO: AVALIAÇÃO DE UMA PROPOSTA PARA PRÉ-ESCOLARES
}

\author{
NUTRITION EDUCATION AS A FORM INTERVENTION: \\ EVALUATION OF A PROPOSAL FOR PRESCHOOLERS
}

\author{
Marcos Coelho BISSOLI ${ }^{1}$ \\ Haydeé Serrão LANZILLOTTI ${ }^{2}$
}

\begin{abstract}
RESUMO
Este estudo teve como objetivo avaliar uma proposta de educação nutricional para pré- escolares em uma creche comunitária. Tal proposta foi formulada a partir de um inquérito dietético, realizado em julho de 1994, que apontou baixa ingestão de calorias, cálcio e ferro por parte das crianças matriculadas na Creche Effatá, localizada no subúrbio do município do Rio de Janeiro. Esta intervenção teve um ano de duração e tem sua estratégia descrita neste trabalho. Para avaliação da proposta foram utilizados dois métodos: um qualitativo e outro quantitativo. O método qualitativo empregado foi a pesquisa participante, analisando-se trës categorias: funcionários da creche, responsáveis pelas crianças e crianças. Para a análise quantitativa optou-se pelo inquérito dietético por pesagem direta de alimentos, comparando-se os resultados com as recomendações da Food Agriculture Organization/World Health Organization. Apesar do tratamento estatístico não mostrar significância na elevação da ingestão dos nutrientes, do ponto de vista educacional, a quantidade consumida é uma questão secundária quando se está trabalhando com formação de atitudes.
\end{abstract}

Termos de indexação: creches, educação nutricional, inquéritos nutricionais, intervenção nutricional, pré-escolar, criança.

\begin{abstract}
This study had the objective of evaluation a nutrition education proposal for preschoolers from a nursery school. This proposal was formulated from a dietary survey - carried out in July, 1994 - that showed low intake of calories, calcium and iron by the children enrolled on the Effatá Nursery School, located in the suburb Rio de Janeiro municipality. This intervention endured one year and its strategy is described in this work. Two methods were used to evaluate the proposal: a qualifying one and a quantifying one. The qualifying

\footnotetext{
1) Bolsista de Extensão, Aluno do Curso de Graduação em Nutrição da Universidade do Estado do Rio de Janeiro, Rua São Francisco Xavier, 524, $12^{\circ}$ andar, Bloco D, 20550-013, Rio de Janeiro, RJ.

(2) Professora do Departamento de Nutrição Social, Instituto de Nutrição da Universidade do Estado do Rio de Janeiro.
} 
method employed was the participant research, with the analysis of the participation of three categories: the nursery school employees, the persons responsible for the children. The dietary inquiry with direct weighing of food was chosen for the quantifying analysism comparing its results with the recomendations of Food Agriculture Organization/World Health Organization. Althoug the statistic survey has not shown significant nutrient intake increase, from the point of view of education the amount consumed is a secondary question when formation of attitudes is the object of the work.

Index terms: nurseries, nutrition education, nutrition surveys, nutrition intervention, preschool, child, nutritionist.

\section{INTRODUÇÃO}

A Educação Nutricional tem por finalidade a formação de atitudes e práticas que conduzam à melhoria da saúde (MOTTA \& BOOG,1991). Por isso seu conceito insere-se no de Educação para a saúde como "qualquer combinação de atividades de informação e educação que leve a uma situação em que as pessoas desejem estar sãs, saibam como alcançar a saúde, façam o que puderem individual e coletivamente para manter a saúde e busquem ajuda quando a necessitem" (CATALÁN et al., 1993). Tal conceito baseia-se nas teorias críticas da educação que têm como principal característica a dependência da educação em relação à sociedade buscando sempre transformar os conhecimentos ao invés de reproduzi-los (SAVIANI, 1991). Fundamentado nessas teorias, VALENTE (1989) defende a educação nutricional crítica, a qual buscou-se empregar na proposta de educação nutricional descrita neste trabalho.

A Educação Nutricional justifica-se como um meio efetivo de produzir mudanças ou alterações nos padrões de comportamento de grupo ou familiar (HAKIM \& SOLIANO, 1989; MOTTA \& BOOG, 1991), ensinando como utilizar os recursos de alimentação fornecidos e até mesmo contribuindo com a adequação de nutrientes na dieta (HUNT et al., 1976; VALENTE, 1989). Deve-se ainda levar em consideração que a Educação Nutricional dirigida a crianças pode contribuir para a formação de hábitos alimentares adequados, uma vez que o comportamento na idade adulta depende do aprendizado recebido na infância (WATSON, 1947; CARTHY, 1969; LUNDIN,1977).

O objetivo desta pesquisa é avaliar a proposta de Educação Nutricional para pré-escolares desenvolvida em uma creche comunitária. A avaliação da propostajustifica-se pela necessidade de quantificar o impacto dessa intervenção nutricional no período de um ano. Tal avaliação possibilita detectar os pontos positivos e negativos da metodologia empregada e reorganizar a forma de intervenção baseando-se nos resultados obtidos.

A Creche Effatá é uma instituição pertencente à Igreja Metodista de Irajá, localizada no subúrbio do município do Rio de Janeiro. Atende, em média, 70 crianças de 2 a 6 anos matriculadas. São oferecidas quatro refeições por dia: desjejum, almoço, lanche e jantar, visando atender $70 \%$ das recomendações nutricionais segundo o padrão da Food and Agriculture Organization e da Organização Mundial da Saúde (FOOD...,1975; ORGANIZACIÓN...,1985). Para tanto, esta creche conta com uma bolsa de alimentação fornecida pela Secretaria Municipal de Desenvolvimento Social.

Os resultados do inquérito dietético por pesagem direta dos alimentos realizado por graduandos do curso de Nutrição da Universidade do Estado do Rio de Janeiro em julho de 1994, concluiu que as crianças ingeriam quantidades inadequadas de calorias, cálcio e ferro. E relevante relatar que durante tal inquérito observaram-se restos de alimentos, os quais foram objeto de correção nos cálculos da ingestão de nutrientes.

\section{MATERIAL E MÉTODOS}

\subsection{Estratégias empregadas no programa edu- cativo}

A partir deste diagnóstico, formulou-se uma proposta de Educação Nutricional com o objetivo de desenvolver nas crianças atitudes adequadas em relação ao alimento, considerando para tanto aspectos cognitivos, emocionais e comportamentais. Com relação aos aspectos cognitivos, atentou-se para a capacidade de abstração ainda em desenvolvimento na faixa etária dos sujeitos. Daí a necessidade de usar 
materiais concretos nas atividades. Quanto aos aspectos emocionais, maior importância foi dedicada à incentivação, utilizando-se materiais multicoloridos a fim de motivá-los a buscar soluções para os problemas a serem apresentados (RAIMAN, 1960; NÉRICI, 1976). Evidentemente, maior ênfase foi dedicada aos nutrientes de baixa ingestão detectados nos diagnósticos.

Não se pôde precisar o tempo gasto em cada atividade, uma vez que o programa educativo se inseriu nas atividades pedagógicas. Podemos garantir que o contato das crianças com a temática da nutrição ocorreu por cinco meses. Todo o processo durou 12 meses, desde o planejamento até a avaliação.

A dinâmica da proposta de trabalho (Figura 1) fundamentou-se na integração entre as atividades de orientação nutricional e pedagógicas não só aos sujeitos de pesquisa (crianças), mas estendeu-se ao nutricionista, aos funcionários da creche e aos responsáveis, como forma de manter o espírito de participação de todos. Baseou-se em experiências concretas (FORRESTER, 1990) onde essa integração é o pressuposto para o sucesso da proposta.

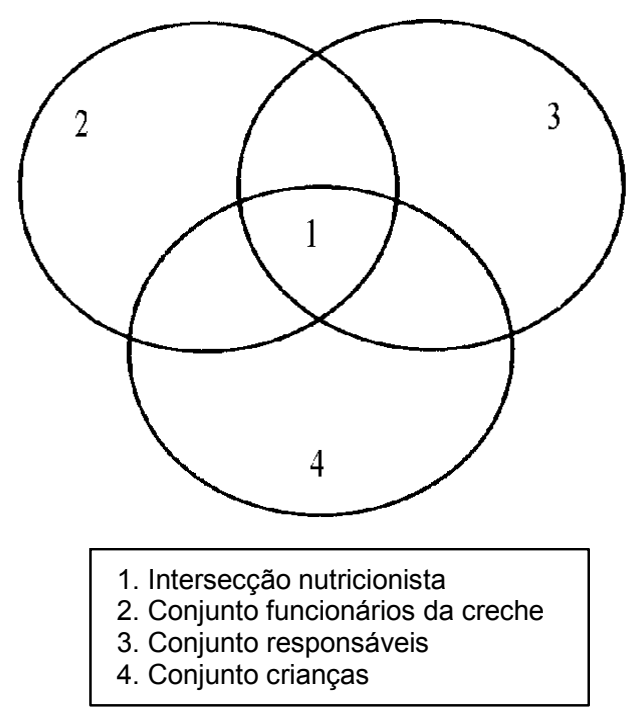

Figura 1. Dinâmica interativa do trabalho.

O nutricionista comparecia à creche de uma a três vezes por semana. Assim, seu tempo de contato as crianças era o mais curto, quando comparados com os outros dois grupos. Tal fato reforça a necessidade destes últimos participarem de forma ativa no desenvolvimento das estratégias educacionais, garantindo a constância do programa. As reuniões de grupos eram realizadas segundo o calendário da creche pelo menos uma por mês.

Neste caso, as estratégias usadas pelo nutricionista em relação aos funcionários prendeu-se a discussões em grupo e leituras dirigidas. Nestas reuniões foram apresentados os resultados do inquérito dietético e explicados os prejuízos advindos da não aquiescência às intervenções propostas. Tais propostas resumiam-se em incentivar as crianças a ingerirem aqueles alimentos mais ricos em nutrientes que se apresentavam deficitários. Em seguida foram traçadas em conjunto, as estratégias que poderiam ser utilizadas com as crianças. Entre várias sugestões, a que pareceu mais adequada, e posteriormente eleita, foi a da criação de uma história com "super-heróis".

A participação dos funcionários da creche na estratégia proposta transpareceu no preparo de materiais a serem utilizados nas diversas atividades. A criação artística dos personagens, concretizando a idéia dos heróis, foi realizada por uma professora de primeiro grau. Novas idéias foram acrescidas à proposta inicial como o lançamento da Semana da Alimentação pela diretora da creche, criação de mural representativo do cardápio do dia por uma das professoras e definição de termos como "amigos da saúde" e "inimigos da saúde".

Reuniões com os responsáveis pelas crianças enriqueceram a proposta de veicular conhecimentos nutricionais. Houve o cuidado, antes mesmo de iniciar as atividades de educação nutricional, de mostrar aos pais todas as etapas da intervenção: diagnóstico antropométrico, diagnóstico dietético e programa educativo. Nestas reuniões preliminares os pais relatavam suas condutas em casa com frases do tipo “...meu filho sempre toma o leite que preparo antes de trazê-Io à creche...”, “... meu filho janta quando chega em casa...”. Tais informações foram úteis na avaliação e reorganização constantes do programa.

Os nutrientes em questão foram apresentados como heróis para as crianças. Personagens "amigos da saúde" começaram a fazer parte do cotidiano das mesmas: "Super" Cálcio, "Super" Ferro e Energia (mais tarde batizada como "Super" Energia pelas funcionárias da creche). Os heróis lutavam, respectivamente, contra os seus "inimigos da saúde": Miudinho (nanismo), Anemia e Bruxa da Fraqueza (desnutrição energético-protéica). 
Os heróis eram utilizados como personagens de histórias, desenhos para serem coloridos e decoração das salas de aula e refeitório. Eram sempre lembrados durante as refeições por funcionários da creche e pelo nutricionista. Assim, os personagens tornaram-se elementos de citação constante durante a permanência das crianças na creche, seja por parte dos funcionários como por parte do nutricionista.

\subsection{Estratégias empregadas para avaliação de resultados}

Para avaliar o impacto da proposta de educação nutricional foram utilizados dois métodos: um método qualitativo e um método quantitativo. $\mathrm{O}$ método qualitativo empregado foi a pesquisa participante. $\mathrm{O}$ método quantitativo foi o inquérito dietético porpesagem direta dos alimentos.

A pesquisa participante, na visão de BORDA (1985), visa não somente à coleta de dados para serem computados, compilados e discutidos, mas sobretudo à ação voltada para as necessidades básicas dos indivíduos, especialmente as classes mais carentes nas estruturas sociais contemporâneas, levando em conta suas aspirações e potencialidades de conhecer, agir e sentir.

Nesta perspectiva, todas as informações coletadas em campo, através da participação ativa do pesquisador, devem ser retornadas à população alvo num processo constante de feedback, comprovando a impossível dissociação entre a pesquisa e a extensão.

A pesquisa qualitativa vem sendo utilizada como solução metodológica alternativa, na tentativa de superar as limitações sentidas no emprego de pesquisas quantitativas em Educação. Nos estudos quantitativos em Educação pode-se correr o risco de submeter o fenômeno educacional a um esquema simplificado de análise, bem como dissociar o sujeito da pesquisa do pesquisador e do seu objeto de estudo (LÜDKE \& ANDRÉ, 1986).

Os estudos dietéticosm exigem análise quantitativa. Contudo, neste caso, o simples fato de uma criança que nunca provou uma carne ou leite - como observado e relatado pelos funcionários da creche - fazê-lo pela primeira vez, pode ser considerado, em análise qualitativa, de extrema relevância. Neste caso importa muito menos a quantidade consumida do que o próprio ato espontâneo de provar o alimento.

Para a avaliação quantitativa, atentou-se em manter a mesma metodologia empregada no inquérito dietético realizado em julho de 1994. Tal conduta justificou-se pela necessidade de comparar os resultados do impacto da proposta de educação nutricional com os resultados anteriores, base informativa para a formulação da mesma.

Optou-se, portanto, pela pesagem direta dos alimentos consumidos, quantifcando-se os alimentos oferecidos às crianças, bem como os restos. Tal método vem sendo apontado como o de maior exatidão (PAO \& CYPEL,1991). É importante salientar que não houveram restos a serem pesados. Portanto, as quantidades de nutrientes ingeridas foram iguais ao porcionamento oferecido.

Para pesagem direta dos alimentos utilizou-se uma balança de prato, com capacidade máxima de $1610 \mathrm{~g}$, com subdivisão de $0,1 \mathrm{~g}$, marca Marte. A medição de líquidos foi feita através de um recipiente de plástico translúcido com volume total de $240 \mathrm{ml}$ e subdivisões de $5 \mathrm{~m} 1$. Os dados foram coletados no período de 8 às 17 horas, incluindo as seguintes refeições: desjejum, almoço, lanche e jantar. A tomada do porcionamento foi obtida através de 12 amostras, sendo 6 para a faixa etária de 2 a 3 anos e 6 para a faixa etária de 4 a 6 anos, obtendo-se os valores em gramas de cada preparação alimentar e alimentos constantes da refeição, incluindo sólidos e líquidos. Os valores médios das porções foram compilados para o cálculo de micro e macro nutrientes da dieta através do Programa de Apoio à Nutrição da Escola Paulista de Medicina ${ }^{1}$. Utilizou-se o parâmetro daFood Agriculture Organization(FAO)/Organização Mundial da Saúde(OMS) para quantificar o percentual de adequação das dietas.

\section{RESULTADOS E DISCUSSÃO}

\subsection{Análise qualitativa}

Os resultados da pesquisa participante demonstraram que a população alvo assimilou favoravelmente a proposta.

\footnotetext{
(3) Dados da tabela de composição química dos alimentos da United States Service Departament of Agriculture, Human-Nutrition - Information

Service: Composition of Foods. (Raw, processed, prepared Agriculture Hand Book n. 8, Series 1-16, Revised 1976-1986).
} 
As crianças conheciam os heróis, identificando-os com suas respectivas imagens e citando as funções de cada herói: “... o Super Cálcio faz crescer...”, “... Energia dá força para brincar...”; identificavam os inimigos de cada herói “... Super Ferro mata o Anemia, né tio?”, “... eu sou o Super Cálcio que pega o Miudinho..."; reconheciam as principais fontes alimentares onde está contido cada herói: “... eu não comia carne, mas agora que eu sei que ela tem Super Ferro, eu como ela todinha..."; sentiam-se motivadas ao alimentar-se pois o alimento estava "... cheio de heróis...".

Além destes relatos, observou-se uma queda dos restos de alimentos tendendo a zero. Tais resultados reforçam a idéia de que educação nutricional é um processo lento e exige persistência.

\subsection{Análise quantitativa}

As Figuras 2 e 3 comparam os resultados do inquérito dietético de julho de 1994 (diagnóstico) com os resultados de julho de 1995 (avaliação), nas faixas etárias de 2 a 3 anos e 4 a 6 anos respectivamente. Embora as Figuras 2 e 3 sugiram o sucesso da intervenção nutricional, o tratamento estatístico demonstrou a não significância na melhoria quantitativa dos níveis de nutrientes ingeridos.

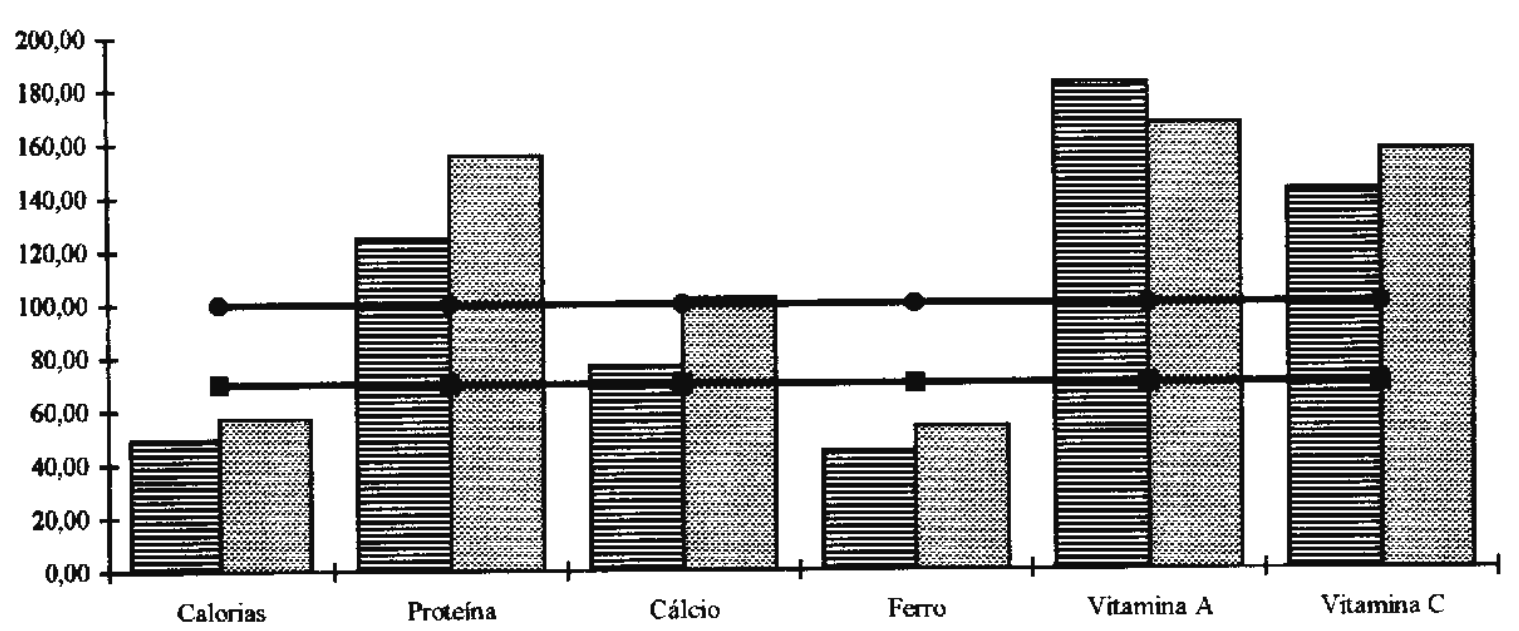

1994 - Reoom. FAO/OMS - Meta Effatá

Figura 2. Confronto da adequação percentual média de energia, proteína, cálcio, ferro, vitamina A e vitamina C nas crianças de 2 a 3 anos da Creche Effatá. em dois inquéritos dietéticos.

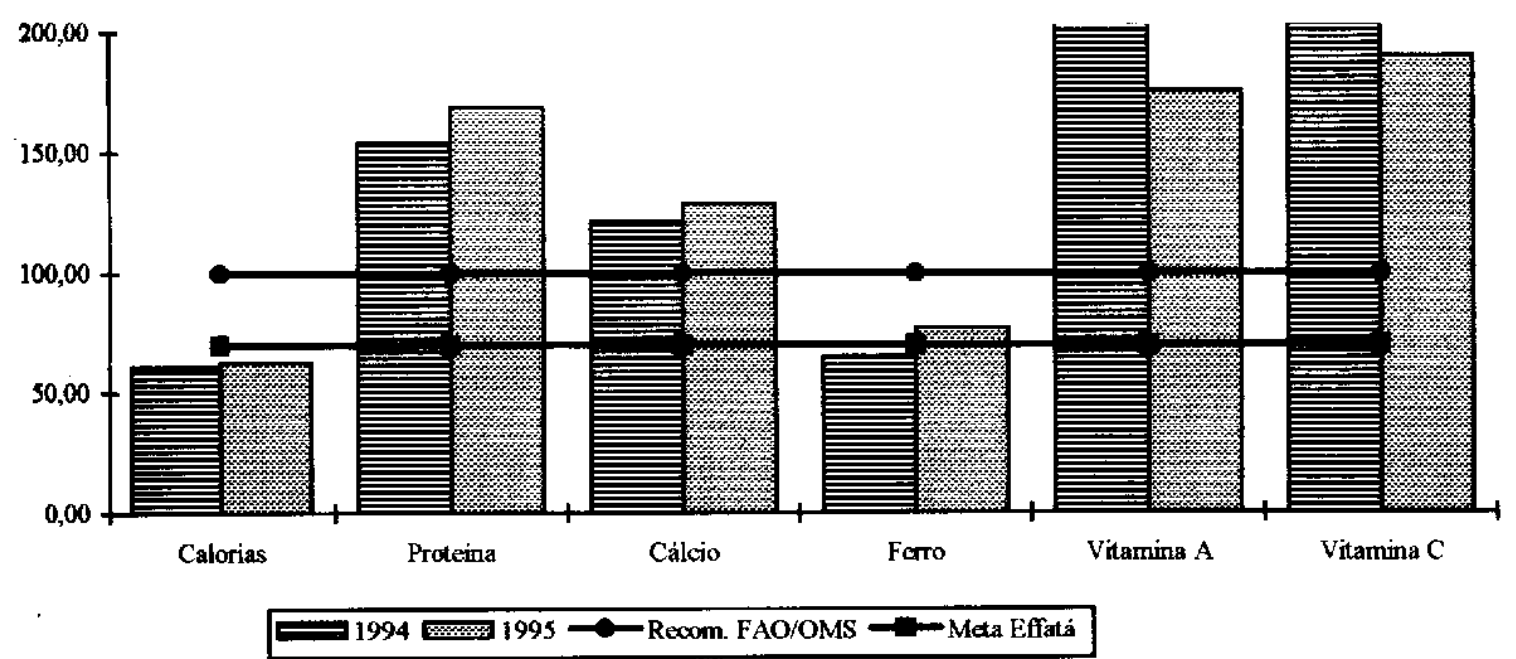

Figura 3. Confronto da adequação percentual média de energia, proteína, cálcio, ferro, vitamina A e vitamina $C$ nas crianças de 4 a 6 anos da Creche Effatá, em dois inquéritos dietéticos. 
Os comitês da FAO e da OMS optaram por utilizar a cifra de mais dois desvios-padrão acima da necessidade média da população (HARPER,1991) para o estabelecimento de recomendações. Este critério se justifica porque recomendações visam a cobrir as necessidades de praticamente todos os indivíduos da população. Entretanto, para calcular a adequação média, pode-se utilizar como valores de referência, o valor da recomendação subtraído de dois desvios-padrão (MONTEIRO \& SZARFARC,1988). Adotando-se este critério, a dieta das crianças da Creche Effatá pode não ser um fator de risco de carência do mineral ferro.

Para o tratamento estatístico, optou-se pela prova unilateral de Kol Mogorov-Smirnov para duas amostras (SIEGEL, 1977), uma vez que se deseja determinar se os valores da população da qual se extraiu uma das amostras são, ou não, estatisticamente maiores do que os valores da população que originou a outra amostra, para testar se houve melhoria do perfll nutricional traduzido pelos inquéritos dietéticos de 1994 e 1995.

$\mathrm{O}$ valor de $\mathrm{Kp}$ do maior desvio na direção prevista calculado pelo software "Statistica: nonparametrics I distributions" não permite dizer da signifcância na melhoria entre os inquéritos dietéticos, logo faz-se necessário mais investimentos em Educação Nutricional (Figuras 4 e 5).

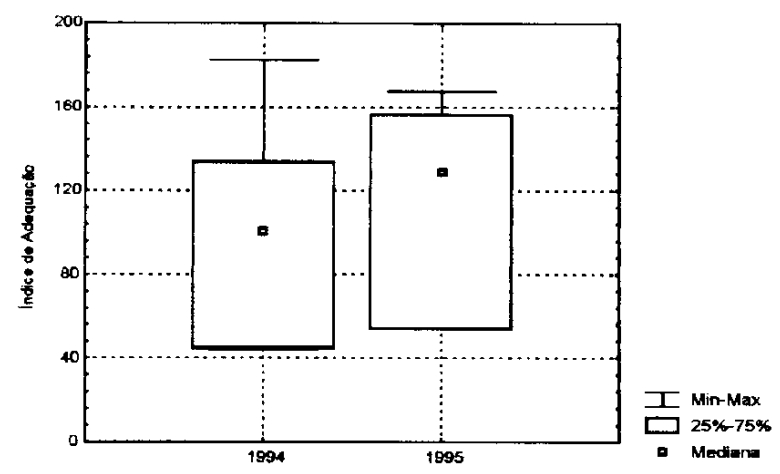

Figura 4. Comparação de inquérito dietético de crianças de 2 a 3 anos

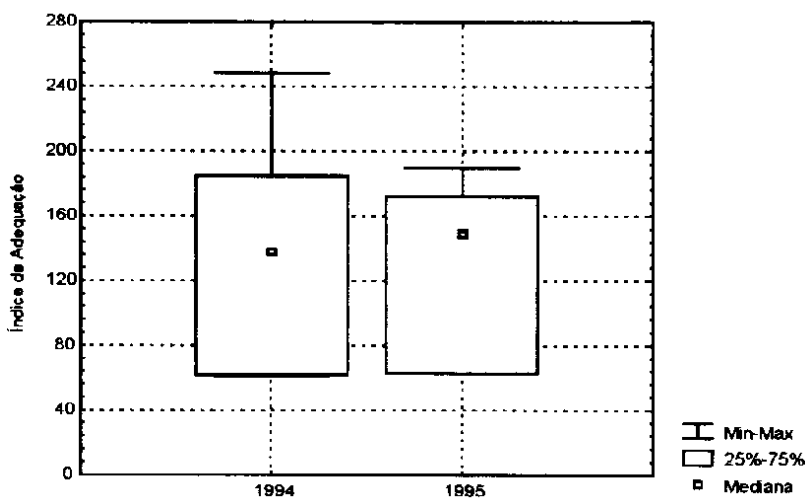

Figura 5. Comparação de inquérito dietético de crianças de 4 a 6 anos.

\section{CONCLUSÃO}

Do exposto, conclui-se que investir em Educação Nutricional pode vir a ser uma forma de intervenção válida, desde que a longo prazo.

É necessário, ainda, buscar uma estratégia adequada aos sujeitos, aos quais a educação nutricional será dirigida. Logo, não se pode prescindir de um diagnóstico prévio.

Este estudo buscou avaliar o sucesso da intervenção nutricional traduzida pela proposta anteriormente descrita. Através da análise qualitativa, percebe-se que a população alvo assimilou favoravelmente os conteúdos. No entanto, a análise quantitativa não permite dizer da significância na melhoria entre os inquéritos dietéticos. Levando-se em consideração que não foram observados restos durante o inquérito dietético, e que as crianças passaram a ingerir alimentos anteriormente rejeitados, pode-se concluir que as estratégias de educação nutricional foram positivas, e que deve-se investir na adequação do porcionamento alimentar oferecido às crianças.

Este processo educacional ainda não se esgotou. Se considerarmos que o processo educativo se constrói no dia-a-dia de cada criança na creche, há motivos suficientes para insistir na proposta eleita. 


\section{REFERENCIAS BIBLIOGRÁFICAS}

BORDA, O.F. Aspectos teóricos da pesquisa participante: considerações sobre o significado e o papel da ciência na participação popular. In: BRANDÃO, C.R. (Org.). Pesquisa participante. 5.ed. São Paulo : Brasiliense, 1985.p.42-62.

CARTHY, J.D. O estudo do comportamento. São Paulo : Companhia Nacional, 1969.109p.

CATALÁN, V.G., SALA, M.J.R., BEGUER, A.C. La educación para la salud: una propuesta fundamentada desde el campo de la docencia. Enseñanza de las ciencias, Barcelona, v.11, n.3, p.289-296, 1993.

FOOD AND AGRICULTUREORGANIZATION. Manual sobre necessidades nutricionais del hombre. Roma, 1975. $198 \mathrm{p}$.

FORRESTER, C. Topics and comments: project lifestyle. Cajanus, Mona, v.23, n.3, p.141-145, 1990.

HAKIM, P., SOLIANO, G. Nutrição e desenvolvimento nacional: estabelecendoa conexão. In:VALENTE, F.L.S. (Coord.). Fome e desnutrição: determinantes sociais. 2.ed. São Paulo: Cortez, 1989. 107p.p.29-47.

HARPER, A.E. Patrones dietéticos y pautas dietéticas. In: INSTITUTO INTERNACIONAL DE CIENCIAS DE LA VIDA. Conocimientos actuales sobre nutrición. 6.ed. Washington DC: OPAS/ILSI, 1991. 614p.p.569-579.

HUNT; I.F., JACOB, M., OSTERGARD, N.J., MASRI, G., CLARK, V.A., COULSON, A.H. Effect of nutrition education on the nutritional status of low-income pregnant women of Mexican discent. American Journal of Clinical Nutrition, Maryland, v.29, n.6, p.675-684, 1976.

LÜDKE, M., ANDRÉ, M.E.D.A. Pesquisa em educação: abordagens qualitativas. São Paulo : EPU, 1986. 99p.
LUNDIN, R.W. Personalidade: uma análise do comportamento. 2.ed. São Paulo: EPU,1977.577p.

MONTEIRO, C.A., SZARFARC, S.C.A. Alimentação. In: MONTEIRO, C.A. Saúde e nutrição das crianças de São Paulo. São Paulo: HUCITEC, 1988.165p.p.71-81.

MOTTA, D.G. da, BOOG, M.C.F. Educação nutricional. 3.ed. São Paulo: IBRASA, 1991. 182p.

NÉRICI, I.G. Didática geral e dinâmica. 2.ed. Rio de Janeiro: Científica,1976.314p.

ORGANIZACIÓN MUNDIAL DE LA SALUD. Necessidades deenergiay de proteinas. Ginebra,1985. 220p.

PAO, E.M., CYPEL, Y.S. Cálculo de la ingesta dietética. In: INSTITUTO INTERNACIONAL DE CIENCIAS DE LA VIDA. Conocimientos actuales sobre nutrición. 6.ed. Washington, DC: OPAS/ILSI, 1991. 614p. p.461-469.

RAIMAN, E.S. Telling is not teaching. Journal of The American Dietetic Association, Chicago, v.37, n.2, p.118-120,1960.

SAVIANI, D. Escola e democracia: teorias da educação, curvatura da vara; onze teses sobre educação e política. 24.ed. São Paulo: Cortez,1991.103p.

SIEGEL, S. Estatistica não-paramétrica para as ciências do comportamento. São Paulo : McGraw-Hill do Brasil, 1977.350p.

VALENTE, F.L.S. (Coord.). Fome e desnutrição: determinantes sociais. 2.ed. São Paulo : Cortez,1989, 107p.p.17-18;66-94.

WATSON, J. El conductismo. Buenos Aires : Paidos,1947. $394 p$.

Recebido para a publicação em 27 de março de 1996 e aceito em 11 de março de 1997. 